\title{
Transcriptome Sequencing Explores the Mechanism of Baicalin on Bone Cancer Pain
}

\author{
Aitao Wang' \\ Dongmei Guo' \\ Hongyu Cheng ${ }^{2}$ \\ Hui Jiang ${ }^{3}$ \\ Xiaojuan Liu' \\ Zhizhong Yun $\mathbb{1 D}^{4}$
}

'Department of Anesthesiology, Inner Mongolia People's Hospital, Hohhot, Inner Mongolia, 0I0017, People's Republic of China; ${ }^{2}$ Department of Anesthesiology, Inner Mongolia Medical University, Hohhot, Inner Mongolia, 0I0I I0, People's Republic of China; ${ }^{3}$ Department of Anesthesiology, Baotou Medical College, Baotou, Inner Mongolia, 014040, People's Republic of China; ${ }^{4}$ Department of Urinary Surgery, Inner Mongolia People's Hospital, Hohhot, Inner Mongolia, OI00I7, People's Republic of China
Correspondence: Zhizhong Yun Department of Urinary Surgery, Inner Mongolia People's Hospital, \#20,

Zhaowuda Road, Saihan District, Hohhot, Inner Mongolia, 010017, People's Republic of China

Tel +86-|8047|9|483

Email yzzybI68@I26.com
Introduction: Bone cancer pain is characterized by persistent pain, usually requiring drugs to relieve pain. Baicalin, a flavonoid compound extracted from Scutellaria baicalensis, which has antioxidant and analgesic effects. But, the effect of baicalin on bone cancer pain is unclear. Thus, this study aimed to explore the mechanism of baicalin on SD rats with bone cancer pain.

Materials and Methods: The MADB-106 breast cancer cells-induced bone pain model was constructed and carried out baicalin treatment. The therapeutic effect of baicalin on bone cancer pain model was observed by hematoxylin-eosin staining and immunofluorescence staining. We also performed transcriptome sequencing analysis of baicalin in the treatment of bone metastases. Also, RT-qPCR and ELISA were used to detect the expression levels of inflammation factors.

Results: After baicalin treatment, osteoclast activation was inhibited and the number of bone trabeculae was increased. Baicalin inhibited the protein expression level of inflammatory factors (IL-1 $\beta$, IL-6, TNF- $\alpha$ and PGE2) in the bone metastases group. Based on the transcriptome sequencing of the bone metastases group and the baicalin treatment group, baicalin inhibited the expression of ALPP, DUSP1, CYR61, ALPPL2, SPP1 and TLR4. RTqPCR was also used to validate the expression levels of these cytokine genes.

Conclusion: Baicalin had a certain inhibitory effect on the SD rat model of bone metastasis cancer. These insights can guide future research on the molecular mechanism of bone cancer pain and provide a theoretical basis for baicalin in the treatment of bone pain caused by breast cancer in the future.

Keywords: breast cancer, bone cancer pain, baicalin, transcriptome sequencing

\section{Introduction}

With the continuous improvement of cancer treatment technology, the 3-year and 5 -year survival rates of cancer patients have been greatly improved. However, cancer still cause pain to the patients, which seriously affects their quality of life. This is because in many common cancers, tumors can easily metastase to the spine, hips, ribs, femur and tibia, ${ }^{1}$ and then invade the surrounding soft tissue. Usually, tumor metastasis to bone will activate osteoclasts and lead to bone resorption, resulting in moderate to severe persistent pain.,3 According to statistics, about $60-90 \%$ of patients with advanced cancer have suffered from varying degrees of pain, of which about $30 \%$ have suffered from continuous severe pain, ${ }^{4}$ which may be related to bone metastasis. As report went, up to $70 \%$ of breast and prostate cancer patients and up to $30 \%$ of thyroid, bladder and lung cancer patients had bone metastases. $^{5}$ 
Currently, the treatment of pain in bone metastasis involved the use of a variety of adjuvant methods, including surgery, radiotherapy, chemotherapy, as well as drug treatment-bisphosphonates, calcitonin, analgesics. Nevertheless, the side effects of some drugs limit their clinical application, such as gastric ulcer and nephrotoxicity of non-steroidal anti-inflammatory analgesics. ${ }^{6}$ In recent years, Chinese herbal extracts have attracted the attention of many researchers. Baicalin (7-d-glucuronic acid, 5,6-dihydroxyflavone) is a flavonoid extracted from Scutellaria baicalensis and other Chinese herbal medicines. Baicalin has anti-tumor, ${ }^{7}$ anti-inflammatory, ${ }^{8}$ neuroprotective ${ }^{9}$ and anti-anxiety effects. ${ }^{10}$ In previous studies, baicalin can significantly inhibit the proliferation of human prostate cancer, ${ }^{11}$ and induce the apoptosis of human colorectal cancer cells SW620. ${ }^{12}$ Also, Wang et al revealed that baicalin suppressed the invasion and metastasis of human osteosarcoma cells by EMT induced by TGF- $\beta 1 .{ }^{13}$ However, the treatment mechanism of baicalin on bone metastases cancer has not been studied yet.

In the study, we explored the therapeutic effect of baicalin on bone metastasis by constructing SD rat model of bone metastasis cancer and carrying out baicalin treatment. And we used transcriptomics sequencing technology and RT-qPCR verification experiments to explore the treatment mechanism of baicalin on bone metastasis cancer. We hope to provide a theoretical basis for baicalin in the treatment of bone metastases $=$ cancer.

\section{Methods}

\section{Cell Culture}

MADB-106 breast cancer cells were purchased from the Beijing Union Medical College Cell Center, and the cells were cultured into DMEM/F12 medium containing 10\% FBS in a $37^{\circ} \mathrm{C} 5 \% \mathrm{CO} 2$ incubator. When the cells reached the logarithmic phase (70-80\%), trypsin was used to digest cells and collected cell suspension. After centrifugation, a cell suspension with a concentration of $1 \times 10^{5}$ cells $/ 10 \mu \mathrm{L}$ final dilution was prepared and put it on ice for standby.

\section{Induction of Bone Cancer Pain and Treatment with Baicalin}

SD rats were randomly divided into the NC group and the Bone metastases group after adaptive feeding for one week. SD rats were anesthetized with pentobarbital sodium $(50 \mathrm{mg} / \mathrm{kg})$. In a simple fixation device, the legs of SD rats were fixed with clips and the left leg was shaved. After disinfection with $70 \% \mathrm{v} / \mathrm{v}$ ethanol, the skin incision was parallel to the tibia. Drill a hole in the left tibial plateau with the disposable blood collection needle, and the breast cancer cell suspension $\left(1 \times 10^{5}\right.$ cells $)$ diluted with $50 \mu \mathrm{L}$ normal saline was slowly injected into the bone marrow cavity by a disposable sterile injection needle. The control group was injected with the same amount of normal saline into the bone marrow cavity. ${ }^{14,15}$ In order to prevent cells from leaking out of the bone, the intraosseous injection hole was closed with bone wax and the tumor cells left outside the bone marrow cavity were removed with $70 \% \mathrm{v} / \mathrm{v}$ ethanol. The animals were placed on a warm mat for recovery and then put in separate cages. During the whole operation, the aseptic operation was strictly followed. In addition, after injection, some SD rats with bone cancer pain were intraperitoneally injected with baicalin $(30 \mathrm{mg} / \mathrm{kg})$ every 2 days for 15 days.

\section{Real-Time Fluorescence Quantitative PCR}

Firstly, total RNA was extracted from the tumor tissues in bone marrow taken at 1 month by Tissues RNA Miniprep Kit (BW-R1-02, Biomiga) and was stored at $-20^{\circ} \mathrm{C}$. cDNA was synthesized by the PrimeScript ${ }^{\mathrm{TM}} \mathrm{RT}$ reagent Kit with gDNA Eraser (AJ51485A, Takara). The amplification reaction was completed by Hieff $^{\circledR}$ qPCR SYBR $^{\circledR}$ Green Master Mix (Low Rox) (H1911331, Shanghai Yisheng Biotechnology Co., Ltd) with the primers listed in Supplementary Table 1. The amplification procedure was performed by pre-denaturation at $50^{\circ} \mathrm{C}$ for $2 \mathrm{~min}$, followed by 40 cycles of $95^{\circ} \mathrm{C} 10 \mathrm{sec}$ and $60^{\circ} \mathrm{C} 30$ sec. The final extension procedure was performed by $95^{\circ} \mathrm{C}$ $15 \mathrm{sec}$. Subsequently, the amplification curve was obtained at $60^{\circ} \mathrm{C} 1 \mathrm{~min}$ and $95^{\circ} \mathrm{C} 15 \mathrm{sec}$. Data were statistically analyzed with $2^{-\Delta \Delta \mathrm{Ct}}$.

\section{Enzyme-Linked Immunosorbent Assay}

The blood sample of SD rats in the control group, the Bone metastases group and Bone metastases + Baicalin group were taken from the eyes of anesthetized mice. Enzyme linked immunosorbent assay (ELISA) was used to measure the protein level of tumor-derived cytokines (PGE2/IL-1 $\beta / \mathrm{IL}-6 / \mathrm{TNF}-\alpha$ ) in mouse serum samples. Mouse derived PGE2 ELISA Kit was purchased from Shanghai renjie biotechnology Co., Ltd. Also, mouse derived ELISA Kit (IL-1 $\beta /$ IL-6/TNF- $\alpha$ ) was purchased 
from Shanghai Biyuntian Biotechnology Co., Ltd. The specific steps were as follows: (1) The whole blood collected from the anesthetized mouse was put into the prepared 96-well ELISA plate, wrapped in an aluminum foil bag and placed at room temperature for $20 \mathrm{~min}$. (2) We set up the standard hole and the sample hole. Add $50 \mu \mathrm{L}$ of different concentrations of the standard, $50 \mu \mathrm{L}$ of sample to be tested into the standard hole and the sample hole, respectively. (3) Then add $100 \mu \mathrm{L}$ of antibody labeled with peroxidase (HRP) into the sample hole and standard hole, seal the orifice plate, and incubate in an incubator for 60 min. (4) Discard the liquid and add the washing solution for multiple washing. (5) Add $50 \mu \mathrm{L}$ of substrate A and B, and incubate at $37^{\circ} \mathrm{C}$ for $15 \mathrm{~min}$. (6) The $\mathrm{OD}$ value of each hole at the wavelength of $450 \mathrm{~nm}$ was detected by Thermo Scientific microplate reader.

\section{Hematoxylin-Eosin Staining}

The tibia of the inoculated side was taken about 22 days after inoculation, and the surrounding muscle soft tissue was stripped and put it into $15 \%$ hydrochloric acid formaldehyde decalcification solution for 3 days. After softening, the tissue was fixed in $70 \%$ ethanol, and then dehydrated, embedded in paraffin, sectioned and stained with hematoxylin-eosin. Tumor growth and bone structure damage were observed under microscope.

\section{Immunofluorescence Staining}

Firstly, the tissue sections were fixed with 4\% paraformaldehyde for $15 \mathrm{~min}$ and permeated with $0.1 \%$ Triton-X 100 for $10 \mathrm{~min}$. The section was incubated with anti-iNOS primary antibody (Santa Cruz Biotechnology, SC-651) overnight. The primary antibody was washed without hybridization by PBS, and then incubated with the fluorescent secondary antibody. Subsequently, the sections were immersed in tartrate resistant acid phosphatase (Trap, Servicebio, G1050) solution and counterstained with hematoxylin dye solution (Servicebio, G1004) to display the nuclei. After multiple washing and drying, anhydrous ethanol and xylene were dehydrated and sealed. Under the optical microscope (Nikon, ECLIPSE E100) and imaging system (Nikon, DS-U3), the tissue sections were examined and analyzed by Imaris 8 image analysis software.

\section{Functional Analyses}

Based on the results of the transcriptome sequencing, Geno ontology (GO) and Kyoto encyclopedia of genes and genomes (KEGG) were used to observe the function of differentially expressed genes (DEGs) presented by Fisher's exact test using the clusterProfiler 2.2.1 version. Biological process (BP), cell composition (CC) and molecular function (MF) were included in GO annotation analysis. KEGG enrichment analysis mainly focused on the mRNAs-related signaling pathways.

\section{Statistical Analysis}

SPSS 20.0 software was used for data management and statistical analysis. The Student's t-tests was used for comparison among three groups. $p$-values obtained from all figures were two-way analysis of variance. All the data were repeated for 3 times. $p$-value $<0.05$ was considered to have significant differences.

\section{Results}

\section{Baicalin Recovered the Bone Resorption in Bone Cancer Pain Rats}

After breast cancer cell injection about 3 weeks, CT was used to examine the degree of tibial destruction in the $\mathrm{NC}$ group, the Bone metastases group and the Bone metastases + Baicalin group, including bone volume fraction (BV/ $\mathrm{TV}$ ), trabecular thickness (Tb.Th), the number of trabeculae (Tb.N) and the mean spacing of trabecular bone (Tb. $\mathrm{Sp}$ ). Compared with the control group, BV/TV, Tb.Th and Tb.N were downregulated in the Bone metastases group. Although the trend of Tb.Sp was opposite, BV/TV, Tb.Th and Tb.N were significantly increased after baicalin treatment (Supplementary Figure 1). These results showed that the bone mineral density (BMD) was reduced and the bone trabeculae was sparse in the Bone metastases group, which proved the success of establishment of cancer pain model from the perspective of radiology. The above results showed that the bone resorption in the Bone metastases group was significantly reduced compared to the $\mathrm{NC}$ group. The bone resorption was recovered after Baicalin treatment (Figure 1).

\section{Baicalin Inhibited Osteoclast Activation and Promoted the Increase of Bone Trabeculae in Bone Cancer Pain Rats}

Besides, the number of trabeculae and osteoclasts in SD rats were also detected in the $\mathrm{NC}$ group, the Bone metastases group and the Bone metastases + Baicalin group detected by HE staining and Trap staining, respectively. Therefore, Trap is a typical marker of osteoclasts, and the results of Trap 


\section{Bone metastases}
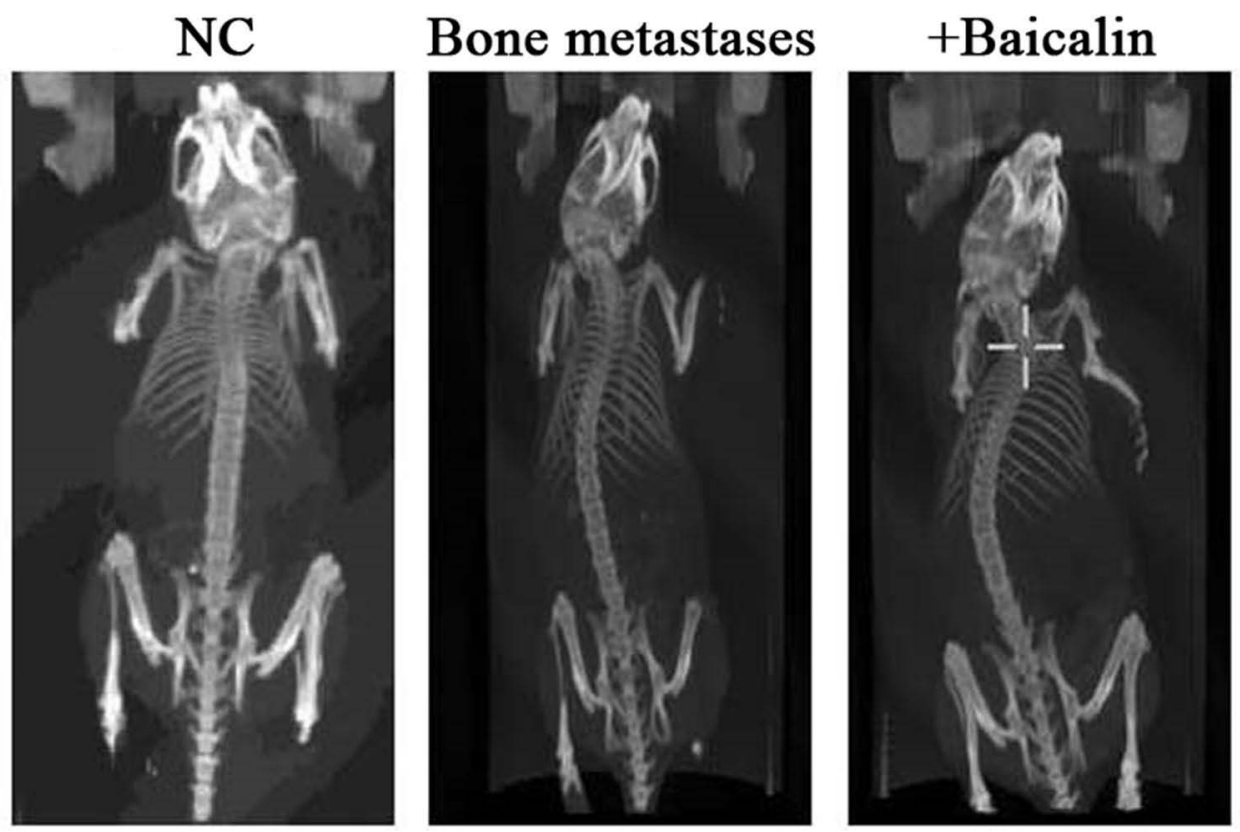

Figure I Baicalin recovered the bone resorption in the Bone metastases cancer. CT was used to detect BMD in SD rats of the NC group, the Bone metastases group and the Bone metastases + Baicalin group. The changes of BMD were calculated by SPSS software.

staining was that the cytoplasm of osteoclasts was wine red and the nucleus was light blue, illustrating that osteoclasts count was significantly increased in the Bone metastases group and was decreased after Baicalin treatment. HE staining also showed that the number of trabeculae decreased in the Bone metastases group and increased in the Bone metastases + Baicalin group (Figure 2).

\section{Baicalin Inhibited the Expression of iNOS in Bone Cancer Pain Rats}

NO has been reported to promote tumor metastasis, and iNOS is one of the four subtypes of NOS, which is the only rate limiting enzyme for NO synthesis. Thus, immunofluorescence detection was utilized to detect the expression of iNOS in the groups. The results revealed that the expression
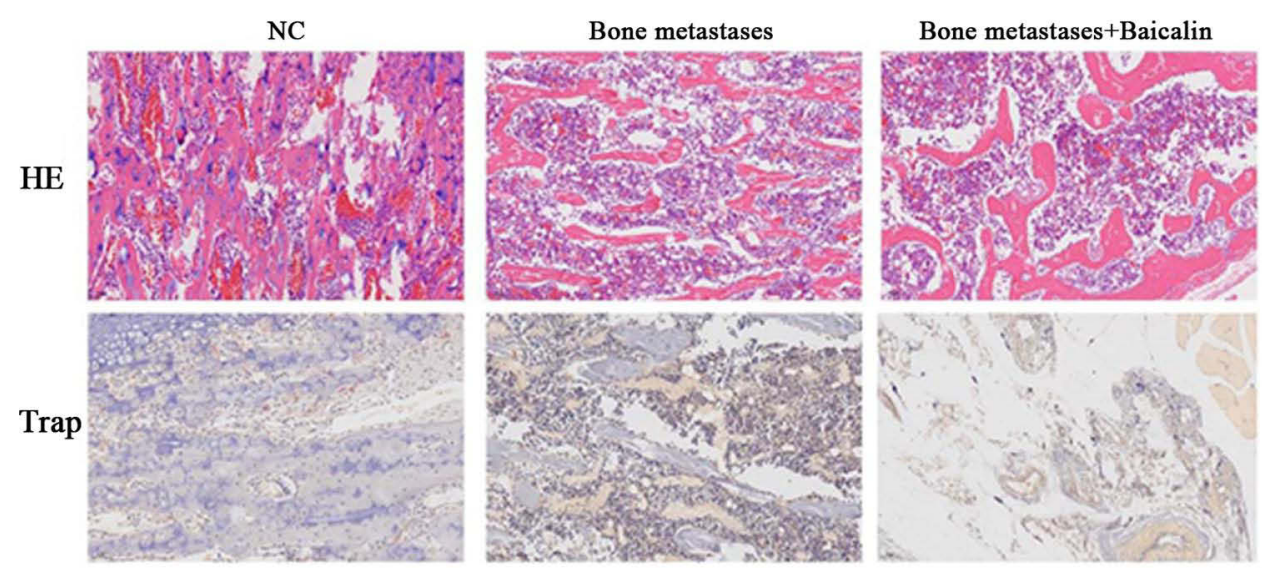

Figure 2 Baicalin inhibited osteoclast activation and promoted the increase of bone trabeculae in the Bone metastases cancer. Trap is a typical marker of osteoclasts, and the results of Trap staining was that the cytoplasm of osteoclasts was wine red and the nucleus was light blue. Trap staining indicated that osteoclasts count was significantly increased in the Bone metastases group and was decreased after Bone metastases + Baicalin. HE staining is one of the commonly used staining methods in paraffin section technology. The nucleus is dyed purple blue and the cytoplasm is dyed pink. HE staining showed that the number of trabeculae decreased in the Bone metastases group and increased after Bone metastases + Baicalin. 
$\mathrm{NC}$

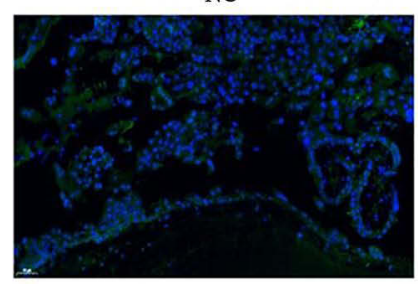

Bone metastases

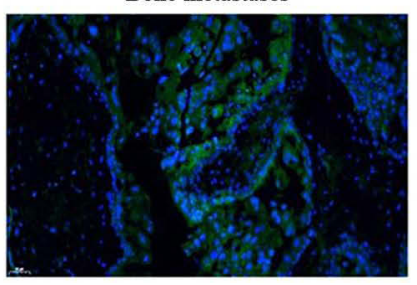

Bone metastases+Baicalin

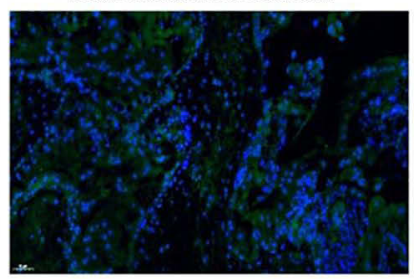

Figure 3 Baicalin inhibited the expression of iNOS in the Bone metastases cancer. iNOS was reported to be related with tumor metastasis. Fluorescein isothiocyanate (FITC) was labeled and showed bright yellow green fluorescence under fluorescence microscope. Immunofluorescence detection revealed that the expression of iNOS was significantly increased in the Bone metastases group and decreased after Bone metastases + Baicalin.

of iNOS was significantly increased in the Bone metastases group and decreased after Baicalin treatment (Figure 3).

\section{Baicalin Inhibited the Expression of Inflammatory Factors in Bone Cancer Pain Rats}

Subsequently, the protein level of inflammatory factors (IL-1 $\beta$, IL-6, TNF- $\alpha$ and PGE2) were examined in the three groups shown by ELISA. In Figure 4 , the IL-1 $\beta$, IL-6, TNF- $\alpha$ and PGE2 protein level in the Bone metastases group was significantly higher than that of in the $\mathrm{NC}$
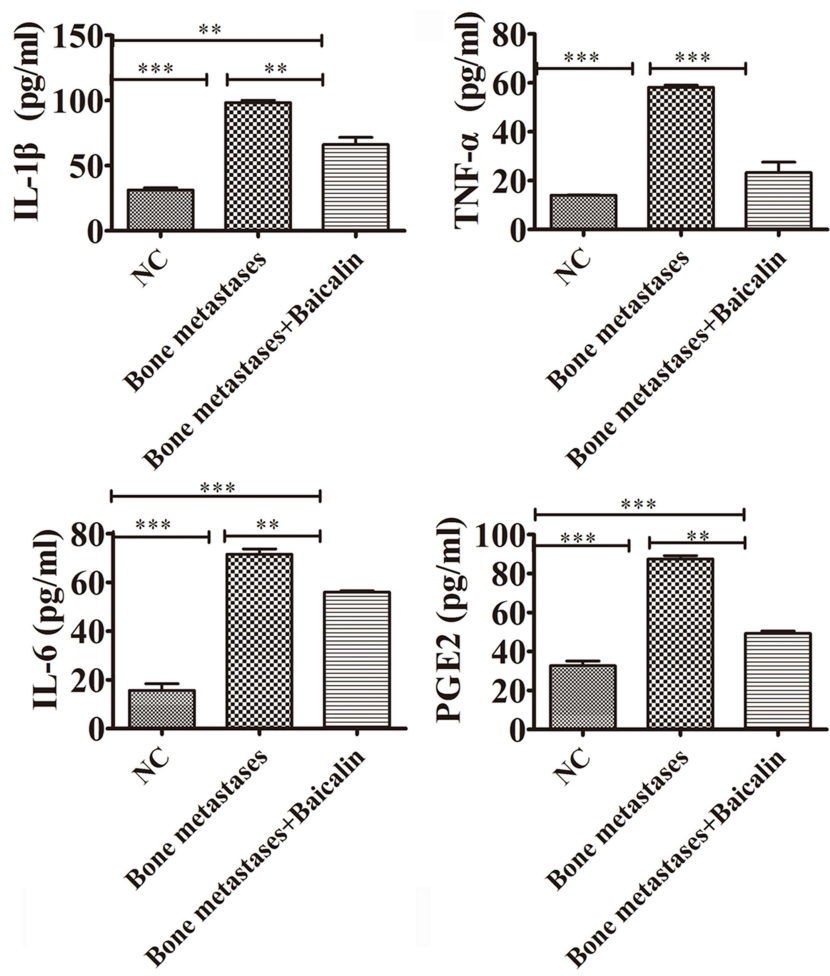

Figure 4 Baicalin inhibited the protein level of inflammatory factors (IL-I $\beta$, IL-6, TNF- $\alpha$ and PGE2) in the Bone metastases cancer shown by ELISA. Data were shown as mean $\pm S D$. $*_{p}<<0.01$, $*_{* *} * 0.001$ was obtained from the NC group vs the Bone metastases group, the NC group vs the Bone metastases + Baicalin group, the Bone metastases group vs the Bone metastases + Baicalin group. group. After Baicalin treatment, IL-1 $\beta$, IL-6, TNF- $\alpha$ and PGE2 expression levels were significantly inhibited.

\section{Transcriptome Sequencing Was Used to Detect the Therapeutic Effect of Baicalin on Bone Cancer Pain}

In order to further study the therapeutic effect of Baicalin on bone cancer pain, we took the spinal cord tissues of SD rats in the NC group, the Bone metastases group and the Bone metastases + Baicalin group for transcriptome sequencing. The GO and KEGG enrichment analysis of DEGs were utilized to study the transcriptome differences in the Bone metastases group vs the NC group (Tables 1 and 2) and the Bone metastases + Baicalin group vs the NC group (Tables 3 and 4). In Tables 1 and 2, the results of top 10 enriched GO pathways of upregulated DEGs showed that the BP changes were in regulation of cellular process, regulation of nitrogen compound metabolic process, ribosome biogenesis, cell cycle, ncRNA processing, response to stimulus, rRNA metabolic process, rRNA processing, organelle organization and positive regulation of cellular metabolic process. Also, the CC changes of DEGs were obviously abundant in nucleus, nucleoplasm, nuclear lumen, intracellular membrane-bounded organelle, cytoplasm, cytosol, mitochondrion and mitochondrial membrane. Changes in MF were mainly enriched in protein binding, protein binding, enzyme binding, metal ion binding, cation binding, catalytic activity, nucleosidetriphosphatase activity and pyrophosphatase activity. The KEGG pathway analysis revealed that the upregulated DEGs were mainly enriched in ribosome biogenesis in eukaryotes, cell cycle, DNA replication, colorectal cancer, pyrimidine metabolism, mismatch repair, biosynthesis of amino acids, estrogen signaling pathway, alanine aspartate and glutamate metabolism, purine metabolism.

In the Tables 3 and 4, the results of top 10 enriched GO pathways of downregulated DEGs displayed that the BP 
Table I Top 10 Enriched GO Pathways of DEGs Between the Bone Metastases Group and the NC Group

\begin{tabular}{|c|c|c|c|}
\hline Terms & Pathway Description & Count & p-value \\
\hline \multicolumn{4}{|l|}{ Upregulated } \\
\hline GO.BP:0050794 & Regulation of cellular process & 411 & $2.09 \mathrm{E}-42$ \\
\hline GO.BP:005।I7I & Regulation of nitrogen compound metabolic process & 270 & $9.39 \mathrm{E}-41$ \\
\hline GO.BP:0042254 & Ribosome biogenesis & 54 & $2.01 \mathrm{E}-34$ \\
\hline GO.BP:0007049 & Cell cycle & 99 & $2.12 \mathrm{E}-29$ \\
\hline GO.BP:0034470 & ncRNA processing & 55 & $6.03 \mathrm{E}-29$ \\
\hline GO.BP:0050896 & Response to stimulus & 299 & $3.40 \mathrm{E}-28$ \\
\hline GO.BP:00I6072 & rRNA metabolic process & 43 & $3.82 \mathrm{E}-28$ \\
\hline GO.BP:0006364 & rRNA processing & 42 & 7.49E-28 \\
\hline GO.BP:0006996 & Organelle organization & 169 & $5.24 \mathrm{E}-26$ \\
\hline GO.BP:003।325 & Positive regulation of cellular metabolic process & 161 & 4.05E-25 \\
\hline GO.CC:0005654 & Nucleoplasm & 234 & $5.65 \mathrm{E}-52$ \\
\hline GO.CC:0043227 & Membrane-bounded organelle & 554 & $3.70 \mathrm{E}-5 \mathrm{I}$ \\
\hline GO.CC:0005737 & Cytoplasm & 487 & I.I2E-50 \\
\hline GO.CC:004323I & Intracellular membrane-bounded organelle & 521 & 6.67E-49 \\
\hline GO.CC:0005829 & Cytosol & 235 & $5.03 \mathrm{E}-34$ \\
\hline GO.CC:0005739 & Mitochondrion & 115 & 8.29E-31 \\
\hline GO.CC:0005740 & Mitochondrial_envelope & 68 & $2.00 \mathrm{E}-23$ \\
\hline GO.CC:0031981 & Nuclear lumen & 271 & $2.96 \mathrm{E}-22$ \\
\hline GO.CC:0031966 & Mitochondrial membrane & 62 & $8.90 \mathrm{E}-21$ \\
\hline GO.CC:0005634 & Nucleus & 378 & 2.0IE-20 \\
\hline GO.MF:00055I5 & Protein binding & 556 & $1.88 \mathrm{E}-76$ \\
\hline GO.MF:0003824 & Catalytic activity & 251 & $5.28 \mathrm{E}-24$ \\
\hline GO.MF:0042802 & Identical protein binding & 104 & $1.18 \mathrm{E}-15$ \\
\hline GO.MF:0003677 & DNA binding & 118 & $2.89 \mathrm{E}-13$ \\
\hline GO.MF:00I9899 & Enzyme binding & 100 & $8.83 \mathrm{E}-10$ \\
\hline GO.MF:0046872 & Metal ion binding & 158 & $8.90 \mathrm{E}-10$ \\
\hline GO.MF:0043I69 & Cation binding & 159 & $3.26 \mathrm{E}-09$ \\
\hline GO.MF:0036094 & Small molecule binding & 107 & 4.37E-09 \\
\hline GO.MF:00I7III & Nucleoside-triphosphatase activity & 47 & $\mathrm{I} .50 \mathrm{E}-08$ \\
\hline GO.MF:0016462 & Pyrophosphatase activity & 49 & $1.92 \mathrm{E}-08$ \\
\hline \multicolumn{4}{|l|}{ Downregulated } \\
\hline GO.BP:0050794 & Regulation of cellular process & 547 & $3.74 \mathrm{E}-65$ \\
\hline GO.BP:0050896 & Response to stimulus & 443 & $6.24 \mathrm{E}-64$ \\
\hline GO.BP:0007I54 & Cell communication & 312 & $5.02 \mathrm{E}-47$ \\
\hline GO.BP:0007I65 & Signal transduction & 294 & $8.60 \mathrm{E}-46$ \\
\hline GO.BP:0023052 & Signaling & 306 & I.36E-45 \\
\hline GO.BP:00I0033 & Response to organic substance & 207 & 3.7|E-4I \\
\hline GO.BP:007I310 & Cellular response to organic substance & 175 & $2.42 \mathrm{E}-38$ \\
\hline GO.BP:0002376 & Immune system process & 189 & 7.67E-36 \\
\hline GO.BP:0032502 & Developmental process & 293 & $2.7 I E-33$ \\
\hline GO.BP:0034097 & Response to cytokine & 107 & $3.91 \mathrm{E}-33$ \\
\hline GO:CC.00I6020 & Membrane & 498 & $5.88 \mathrm{E}-68$ \\
\hline GO.CC:0005737 & Cytoplasm & 596 & 4.77E-52 \\
\hline GO.CC:00I 2505 & Endomembrane_system & 292 & $3.58 \mathrm{E}-50$ \\
\hline GO.CC:0031982 & Vesicle & 270 & $1.08 \mathrm{E}-49$ \\
\hline GO.CC:003I 224 & Intrinsic component of membrane & 318 & 2.IIE-45 \\
\hline GO.CC:0071944 & Cell periphery & 327 & $2.48 \mathrm{E}-44$ \\
\hline GO.CC:0005886 & Plasma membrane & 317 & I.46E-4I \\
\hline
\end{tabular}

(Continued) 
Table I (Continued).

\begin{tabular}{|c|c|c|c|}
\hline Terms & Pathway Description & Count & $p$-value \\
\hline GO.CC:001602I & Integral component of membrane & 302 & $9.88 \mathrm{E}-4 \mathrm{I}$ \\
\hline GO.CC:0005576 & Extracellular region & 274 & $1.68 \mathrm{E}-39$ \\
\hline GO.CC:00056I5 & Extracellular space & 232 & I.39E-38 \\
\hline GO.MF:00055I5 & Protein binding & 680 & I.57E-76 \\
\hline GO.MF:0003824 & Catalytic activity & 297 & 5.97E-22 \\
\hline GO.MF:0005I02 & Signaling receptor binding & 116 & $4.58 \mathrm{E}-17$ \\
\hline GO.MF:0042802 & Identical protein binding & 120 & I.84E-14 \\
\hline GO.MF:00I9899 & Enzyme binding & 132 & $1.81 \mathrm{E}-13$ \\
\hline GO.MF:00I6787 & Hydrolase activity & 144 & $5.36 \mathrm{E}-13$ \\
\hline GO.MF:0030234 & Enzyme regulator activity & 78 & $6.73 \mathrm{E}-13$ \\
\hline GO.MF:0046872 & Metal ion binding & 194 & 4.64E- 10 \\
\hline GO.MF:0043I69 & Cation binding & 198 & 4.73E-10 \\
\hline GO.MF:0022857 & Transmembrane transporter activity & 72 & $7.36 \mathrm{E}-10$ \\
\hline
\end{tabular}

Abbreviations: BP, biological process; CC, cellular component; MF, molecular function; DEGs, differentially expressed genes.

changes were in regulation of cellular process, regulation of nitrogen compound metabolic process, cell cycle, mitotic cell cycle, regulation of cell cycle, positive regulation of cellular metabolic process, regulation of RNA metabolic process, regulation of nucleic acid-templated transcription, regulation of transcription, regulation of RNA biosynthetic process. CC changes were enriched in cytoplasm, cytosol, nucleoplasm, membrane, membrane-bounded organelle,

Table 2 Top 10 Enriched KEGG Pathways of DEGs Between the Bone Metastases Group and the NC Group

\begin{tabular}{|c|c|c|c|}
\hline ID & Pathway Description & Count & p-value \\
\hline \multicolumn{4}{|c|}{ Upregulated } \\
\hline hsa03008 & Ribosome biogenesis in eukaryotes & 15 & I.30E-05 \\
\hline hsa04II0 & Cell cycle & 14 & 2.49E-04 \\
\hline hsa03030 & DNA replication & 7 & $3.50 \mathrm{E}-04$ \\
\hline hsa05210 & Colorectal cancer & 11 & $3.90 \mathrm{E}-04$ \\
\hline hsa00240 & Pyrimidine metabolism & 8 & I.3IE-03 \\
\hline hsa03430 & Mismatch repair & 5 & I.48E-03 \\
\hline hsa0I 230 & Biosynthesis of amino acids & 9 & 2.06E-03 \\
\hline hsa049I5 & Estrogen signaling pathway & 13 & 2.IIE-03 \\
\hline hsa00250 & Alanine aspartate and glutamate metabolism & 6 & $2.49 \mathrm{E}-03$ \\
\hline hsa00230 & Purine metabolism & 12 & $3.64 \mathrm{E}-03$ \\
\hline \multicolumn{4}{|c|}{ Downregulated } \\
\hline hsa04657 & IL-I7 signaling pathway & 21 & $2.69 \mathrm{E}-09$ \\
\hline hsa04668 & TNF signaling pathway & 21 & $7.18 \mathrm{E}-08$ \\
\hline hsa05I34 & Legionellosis & 14 & $3.6 \mathrm{IE}-07$ \\
\hline hsa05I64 & Influenza A & 25 & $6.4 \mathrm{IE}-07$ \\
\hline hsa05I33 & Pertussis & 13 & $6.34 \mathrm{E}-05$ \\
\hline hsa05165 & Human papillomavirus infection & 32 & I.37E-04 \\
\hline hsa05323 & Rheumatoid arthritis & 14 & I.4IE-04 \\
\hline hsa05I45 & Toxoplasmosis & 15 & 3.IIE-04 \\
\hline hsa04060 & Cytokine-cytokine receptor interaction & 28 & 4.84E-04 \\
\hline hsa04210 & Apoptosis & 16 & 8.62E-04 \\
\hline
\end{tabular}

Abbreviation: DEGs, differentially expressed genes. 
Table 3 Top 10 Enriched GO Pathways of DEGs Between the Baicalin Treatment Group and the NC Group

\begin{tabular}{|c|c|c|c|}
\hline Terms & Pathway Description & Count & p-value \\
\hline \multicolumn{4}{|l|}{ Upregulated } \\
\hline GO.BP:0050794 & Regulation of cellular process & 167 & $1.21 \mathrm{E}-18$ \\
\hline GO.BP:0050896 & Response to stimulus & 127 & $5.94 \mathrm{E}-15$ \\
\hline GO.BP:002305I & Regulation of signaling & 70 & $4.99 \mathrm{E}-13$ \\
\hline GO.BP:005II7I & Regulation of nitrogen compound metabolic process & 96 & I.49E-II \\
\hline GO.BP:0009966 & Regulation of signal transduction & 62 & I.83E-II \\
\hline GO.BP:0009968 & Negative regulation of signal transduction & 37 & $6.02 \mathrm{E}-\mathrm{II}$ \\
\hline GO.BP:0009893 & Positive regulation of metabolic process & 72 & $2.40 \mathrm{E}-10$ \\
\hline GO.BP:0032502 & Developmental process & 89 & $3.97 \mathrm{E}-10$ \\
\hline GO.BP:0050790 & Regulation of catalytic activity & 50 & $5.13 \mathrm{E}-10$ \\
\hline GO.BP:0007275 & Multicellular organism development & 79 & $7.73 \mathrm{E}-10$ \\
\hline GO.CC:0016020 & Membrane & 149 & $3.23 \mathrm{E}-18$ \\
\hline GO.CC:0005737 & Cytoplasm & 189 & $9.24 \mathrm{E}-18$ \\
\hline GO.CC:0043227 & Membrane-bounded organelle & 210 & $9.24 \mathrm{E}-15$ \\
\hline GO.CC:00I 2505 & Endomembrane system & 87 & $3.62 \mathrm{E}-\mathrm{I} 4$ \\
\hline GO.CC:0031982 & Vesicle & 75 & I.29E-II \\
\hline GO.CC:004323I & Intracellular membrane-bounded organelle & 188 & 7.27E-II \\
\hline GO.CC:0005576 & Extracellular region & 76 & 4.87E-09 \\
\hline GO.CC:0031090 & Organelle membrane & 62 & $1.90 \mathrm{E}-08$ \\
\hline GO.CC:007I944 & Cell periphery & 86 & 4.09E-08 \\
\hline GO.CC:0099503 & Secretory vesicle & 28 & 4. $14 \mathrm{E}-08$ \\
\hline GO.MF:00055I5 & Protein_binding & 236 & $3.12 \mathrm{E}-37$ \\
\hline GO.MF:0003824 & Catalytic_activity & 105 & 2.7IE-II \\
\hline GO.MF:0042802 & Identical_protein_binding & 46 & $\mathrm{I} .2 \mathrm{IE}-08$ \\
\hline GO.MF:0043169 & Cation binding & 68 & $2.8 \mathrm{IE}-05$ \\
\hline GO.MF:0004857 & Enzyme inhibitor activity & 14 & $2.85 \mathrm{E}-05$ \\
\hline GO.MF:00I6740 & Transferase activity & 43 & 4.70E-05 \\
\hline GO.MF:0046872 & Metal ion binding & 65 & 7.93E-05 \\
\hline GO.MF:0030234 & Enzyme regulator activity & 24 & I.35E-04 \\
\hline GO.MF:0008427 & Calcium-dependent protein kinase inhibitor activity & 2 & 2.73E-04 \\
\hline GO.MF:0008454 & Alpha-1,3-mannosylglycoprotein 4-beta- $\mathrm{N}$-acetylglucosaminyltransferase activity & 2 & $2.73 \mathrm{E}-04$ \\
\hline \multicolumn{4}{|l|}{ Downregulated } \\
\hline GO.BP:0050794 & Regulation of cellular process & 208 & $3.04 \mathrm{E}-28$ \\
\hline GO.BP:005II7I & Regulation of nitrogen compound metabolic process & $|2|$ & $5.28 \mathrm{E}-17$ \\
\hline GO.BP:0007049 & Cell cycle & 49 & $4.65 \mathrm{E}-16$ \\
\hline GO.BP:0000278 & Mitotic cell cycle & 35 & $1.28 \mathrm{E}-15$ \\
\hline GO.BP:005I726 & Regulation of cell cycle & 45 & $1.70 \mathrm{E}-14$ \\
\hline GO.BP:003। 325 & Positive regulation of cellular metabolic process & 78 & $1.38 \mathrm{E}-13$ \\
\hline GO.BP:005I 252 & Regulation of RNA metabolic process & 84 & $3.03 \mathrm{E}-13$ \\
\hline GO.BP:1903506 & Regulation of_nucleic_acid-templated_transcription & 80 & $3.24 \mathrm{E}-13$ \\
\hline GO.BP:0006355 & Regulation of transcription, DNA-templated & 79 & $3.49 \mathrm{E}-13$ \\
\hline GO.BP:200II4I & Regulation of RNA biosynthetic_process & 80 & $3.53 \mathrm{E}-13$ \\
\hline GO.CC:0005737 & Cytoplasm & 223 & $5.24 \mathrm{E}-22$ \\
\hline GO.CC:0005829 & Cytosol & 114 & $1.39 \mathrm{E}-18$ \\
\hline GO.CC:0043227 & Membrane-bounded organelle & 242 & $5.54 \mathrm{E}-16$ \\
\hline GO.CC:0005654 & Nucleoplasm & 87 & $2.43 \mathrm{E}-13$ \\
\hline GO.CC:004323I & Intracellular membrane-bounded organelle & 216 & I.69E-II \\
\hline GO.CC:0016020 & Membrane & 147 & $1.47 \mathrm{E}-10$ \\
\hline GO.CC:00I 2505 & Endomembrane system & 86 & $6.92 \mathrm{E}-10$ \\
\hline
\end{tabular}

(Continued) 
Table 3 (Continued).

\begin{tabular}{|l|l|c|c|}
\hline Terms & Pathway Description & Count & p-value \\
\hline GO.CC:0005856 & Cytoskeleton & 52 & $6.85 \mathrm{E}-09$ \\
GO.CC:0015630 & Microtubule cytoskeleton & 36 & $8.45 \mathrm{E}-09$ \\
GO.CC:0031090 & Organelle membrane & 68 & $4.64 \mathrm{E}-08$ \\
GO.MF:00055I5 & Protein binding & 259 & $4.97 \mathrm{E}-35$ \\
GO.MF:0046872 & Metal ion binding & 82 & $1.24 \mathrm{E}-07$ \\
GO.MF:0043169 & Cation binding & 82 & $3.80 \mathrm{E}-07$ \\
GO.MF:0003824 & Catalytic activity & 98 & $7.76 \mathrm{E}-06$ \\
GO.MF:0140II0 & Transcription regulator activity & $4 \mathrm{I}$ & $3.1 \mathrm{EE}-05$ \\
GO.MF:0045294 & Alpha-catenin binding & 3 & $1.46 \mathrm{E}-04$ \\
GO.MF:0008092 & Cytoskeletal protein binding & 24 & $2.58 \mathrm{E}-04$ \\
GO.MF:0003677 & DNA binding & 47 & $2.64 \mathrm{E}-04$ \\
GO.MF:0030332 & Cyclin binding & 4 & $3.05 \mathrm{E}-04$ \\
GO.MF:0030234 & Enzyme regulator activity & 25 & $3.87 \mathrm{E}-04$ \\
\hline
\end{tabular}

Abbreviations: BP, biological process; CC, cellular component; MF, molecular function; DEGs, differentially expressed genes.

endomembrane system, cytoskeleton and organelle membrane. The MF changes were mainly enriched in protein binding, metal ion binding, cation binding, alpha-catenin binding, cytoskeletal protein binding, DNA binding, cyclin binding, catalytic activity, transcription regulator activity and enzyme regulator activity. As for the top $10 \mathrm{KEGG}$ KEGG pathway enrichment, the mRNAs were mainly enriched in AGE-RAGE signaling pathway in diabetic

Table 4 Top 10 Enriched KEGG Pathways of DEGs Between the Baicalin Treatment Group and the NC Group

\begin{tabular}{|c|c|c|c|}
\hline ID & Pathway Description & Count & $p$-value \\
\hline \multicolumn{4}{|c|}{ Upregulated } \\
\hline hsa04640 & Hematopoietic cell lineage & 6 & 0.005 \\
\hline hsa05205 & Proteoglycans in cancer & 9 & 0.006 \\
\hline hsa05I45 & Toxoplasmosis & 6 & 0.009 \\
\hline hsa046I0 & Complement and coagulation cascades & 5 & 0.011 \\
\hline hsa0IIO0 & Metabolic pathways & 32 & 0.045 \\
\hline hsa00270 & Cysteine and methionine metabolism & 3 & 0.046 \\
\hline hsa00330 & Arginine and proline metabolism & 3 & 0.046 \\
\hline hsa005I0 & $\mathrm{N}$-Glycan biosynthesis & 3 & 0.046 \\
\hline hsa05323 & Rheumatoid arthritis & 4 & 0.062 \\
\hline hsa00790 & Folate biosynthesis & 2 & 0.064 \\
\hline \multicolumn{4}{|c|}{ Downregulated } \\
\hline hsa04933 & AGE-RAGE signaling pathway in diabetic complications & 7 & 0.001 \\
\hline hsa05168 & Herpes simplex virus I infection & 17 & 0.001 \\
\hline hsa05202 & Transcriptional misregulation in cancer & 9 & 0.003 \\
\hline hsa05I32 & Salmonella infection & 10 & 0.005 \\
\hline hsa04064 & NF-kappa B signaling pathway & 6 & 0.005 \\
\hline hsa04I44 & Endocytosis & 10 & 0.005 \\
\hline hsa05I 44 & Malaria & 4 & 0.007 \\
\hline hsa04668 & TNF signaling pathway & 6 & 0.007 \\
\hline hsa05020 & Prion disease & 10 & 0.009 \\
\hline hsa04218 & Cellular senescence & 7 & 0.010 \\
\hline
\end{tabular}

Abbreviation: DEGs, differentially expressed genes. 

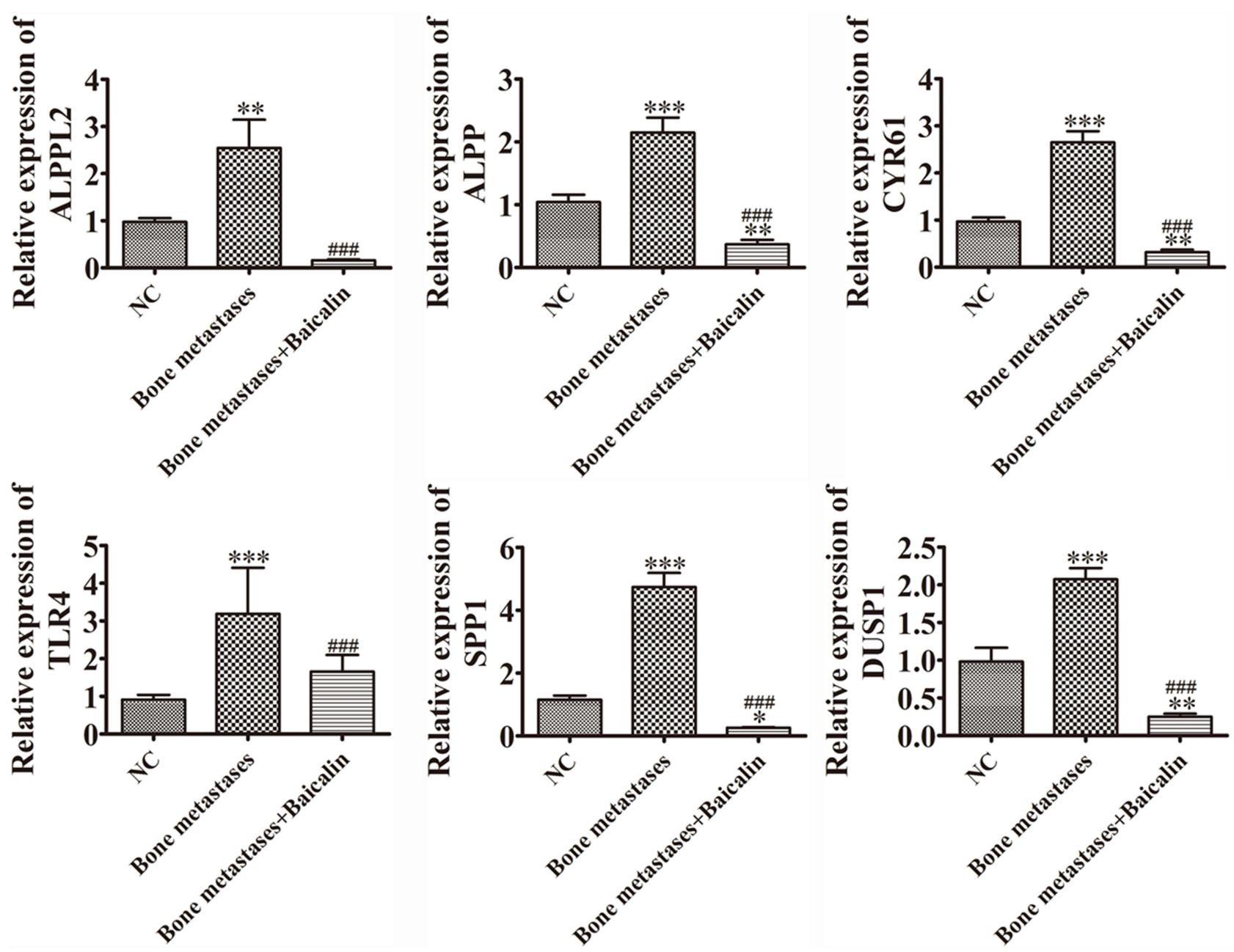

Figure 5 RT-qPCR results indicated that the mRNA expression level of ALPP, DUSPI, CYR6I, ALPPL2, SPPI and TLR4 were significantly up-regulated in the Bone metastases group compared with the NC group. By comparing the Bone metastases + Baicalin group and the NC group, the expression of these genes were significantly downregulated. The RT-qPCR results of these genes were consistent with the results of the transcriptomic sequencing results. Data were shown as mean \pm SD. $* p<0.05$, $* * p<$

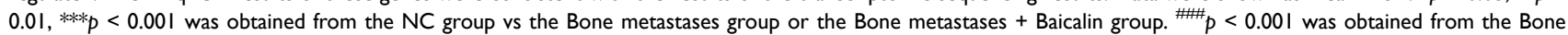
metastases group vs the Bone metastases + Baicalin group.

complications, herpes simplex virus 1 infection, salmonella infection, prion disease, malaria, endocytosis, cellular senescence, transcriptional misregulation in cancer, NF-kappa B signaling pathway, TNF signaling pathway.

\section{RT-qPCR Was Used to Verify the Sequencing Results}

According to the transcriptomic sequencing results, the mRNA expression levels of ALPP, DUSP1, CYR61, ALPPL2, SPP1 and TLR4 were significantly up-regulated in the Bone metastases group compared with the $\mathrm{NC}$ group. By comparing the Bone metastases + Baicalin group and the NC group, the protein expression level of these genes was significantly down-regulated. The RT-
qPCR results of these genes were consistent with the results obtained by transcriptomic sequencing (Figure 5).

\section{Discussion}

It has been reported that the development of bone cancer pain was accompanied by the expression of many genes in peripheral and central nervous system were changed. ${ }^{16-19}$ Some researchers also found the changes of bone cancer pain gene expression profile by transcriptome sequencing. Some genes for the progression of pain hypersensitivity have also been discovered. However, the specific mechanism is still not very clear. In the study, we constructed the SD rat model of bone metastasis induced by MADB-106 cells and treated with baicalin. Based on the transcriptome 
sequencing results and RT-qPCR validation experiments, we found that baicalin had a certain inhibitory effect on the SD rat model of bone metastasis cancer.

Natural products of plants were said to treat chronic diseases, such as pain. ${ }^{20}$ Baicalin is a flavonoid extracted from Huang Qin, which has a variety of physiological functions. It has antioxidant properties, ${ }^{21}$ and has analgesic effect on migraine induced by nitroglycerin in rats, ${ }^{22}$ neuropathic pain in rats with spinal nerve ligation. ${ }^{23,24}$ Also, it was reported that it possessed anti-tumor effect in osteophilic breast cancer through inducing cell apoptosis. ${ }^{25}$ However, the presumptive effect of baicalin on bone cancer pain and its underlying mechanism were not yet clear. In the latest research, Li et $\mathrm{al}^{26}$ found that baicalin can improve mechanical hyperalgesia and thermal hyperalgesia in SD rats with bone cancer pain. In chicken liver inflammation induced by lipopolysaccharide, Cheng et $\mathrm{al}^{27}$ reported that baicalin can inhibit the expression of iNOS by TLR4-Mediated NF-kB Pathway. Min et $\mathrm{al}^{28}$ revealed that baicalin suppressed the higher expression level of iNOS in the process of UVB-induced inflammatory injury by TLR4 pathway. The above results showed that baicalin may regress the expression of iNOS in the process of inflammatory response by TLR4 pathway or TLR4-mediated pathway. In this study, baicalin can inhibit the expression of iNOS in the SD rat model of bone metastasis cancer. In the follow-up, we still need to further explore what pathway baicalin plays a role. Additionally, the results showed that baicalin recovered the bone mineral density and the number of bone trabeculae in the SD rat with bone cancer pain, and inhibited the activation of osteoclasts. On the basis of the transcriptome sequencing results, the expression levels of some inflammatory cytokines were significantly decreased after baicalin treatment of bone cancer pain model. The results illustrated that baicalin had a certain inhibitory effect on the rat model of bone metastasis cancer. These insights can guide future research on the molecular mechanism of bone cancer pain, lay a theoretical foundation for new molecular markers related to pain response, and provide a theoretical basis for baicalin in the treatment of bone pain caused by breast cancer in the future.

\section{Ethics Approval and Consent to Participate}

All experimental procedures have been approved by the Ethics Committee of People's Hospital of Inner Mongolia (202021009L). Animal treatment was performed according to the guidelines of the International Association for pain research. $^{29}$

\section{Acknowledgments}

We thank all participants in the study.

\section{Funding}

This work was supported by the mechanism of Baicalin inhibiting TLR-4 mediated central immune response to alleviate bone cancer pain (2019GG124).

\section{Disclosure}

The authors state that they have no conflicts of interest.

\section{References}

1. Coleman RE. Clinical features of metastatic bone disease and risk of skeletal morbidity. Clin Cancer Res. 2006;12(20):6243s-6249s. doi:10.1158/1078-0432.CCR-06-0931

2. Jung Koo H, Sohn EH, Kim YJ, Jang SA, Namkoong S, Chan Kang S. Effect of the combinatory mixture of Rubus coreanus miquel and astragalus membranaceus bunge extracts on ovariectomy-induced osteoporosis in mice and anti-RANK signaling effect. J Ethnopharmacol. 2014;151:951-959. doi:10.1016/j.jep.2013.12.008

3. Zhu XC, Zhang JL, Ge CT, et al. Advances in cancer pain from bone metastasis. Drug Des Devel Ther. 2015;9:4239-4245.

4. Turabi A, Plunkett AR. The application of genomic and molecular data in the treatment of chronic cancer pain. J Surg Oncol. 2012;105:494-501. doi:10.1002/jso.21707

5. Guerra Liberal FDC, Tavares AAS, Tavares J. Palliative treatment of metastatic bone pain with radiopharmaceuticals: a perspective beyond Strontium-89 and Samarium-153. Appl Radiat Isot. 2016;110:87-99. doi:10.1016/j.apradiso.2016.01.003

6. Pacharinsak C, Beitz A. Animal models of cancer pain. Comp Med. 2008;58:220-233.

7. Zhou QM, Wang S, Zhang H, et al. The combination of baicalin and baicalein enhances apoptosis via the ERK/p38 MAPK pathway in human breast cancer cells. Acta Pharmacol Sin. 2009;30:1648-1658. doi:10.1038/aps.2009.166

8. Jung MA, Jang SE, Hong SW, Hana MJ, Kim DH. The role of intestinal microflora in anti-inflammatory effect of baicalin in mice. Biomol Ther. 2012;20:36-42. doi:10.4062/biomolther.2012.20.1.036

9. Liu YF, Gao F, Li XW, et al. The anticonvulsant and neuroprotective effects of baicalin on pilocarpine-induced epileptic model in rats. Neurochem Res. 2012;37:1670-1680. doi:10.1007/s11064-012-0771-8

10. Huynh DL, Ngau TH, Nguyen NH, Tran GB, Nguyen CT. Potential therapeutic and pharmacological effects of wogonin: an updated review. Mol Biol Rep. 2020;47:9779-9789. doi:10.1007/s11033-020-05972-9

11. Yu Z, Zhan C, Du H, Zhang L, Liang C, Zhang L. Baicalin suppresses the cell cycle progression and proliferation of prostate cancer cells through the CDK6/FOXM1 axis. Mol Cell Biochem. 2020;469 (1-2):169-178. doi:10.1007/s11010-020-03739-1

12. Chen WC, Kuo TH, Tzeng YS, Tsai YC. Baicalin induces apoptosis in SW620 human colorectal carcinoma cells in vitro and suppresses tumor growth in vivo. Molecules. 2012;17:3844-3857. doi:10.3390/ molecules 17043844

13. Wang Y, Wang H, Zhou R, et al. Baicalin inhibits human osteosarcoma cells invasion, metastasis, and anoikis resistance by suppressing the transforming growth factor- $\beta 1$-induced epithelial-tomesenchymal transition. Anticancer Drugs. 2017;28:581-587. doi:10.1097/CAD.0000000000000495

14. Zhang J, Wang LS, Ye SL, Luo P, Wang BL. Blockage of tropomyosin receptor kinase a (TrkA) enhances chemo-sensitivity in breast cancer cells and inhibits metastasis in vivo. Int J Clin Exp Med. 2015;8:634-641. 
15. Linher-Melville $\mathrm{K}$, Sharma M, Nakhla $\mathrm{P}$, et al. Inhibiting STAT3 in a murine model of human breast cancer-induced bone pain delays the onset of nociception. Mol Pain. 2019;15:1744806918823477. doi:10.1177/1744806918823477

16. Hu XF, He XT, Zhou KX, et al. The analgesic effects of triptolide in the bone cancer pain rats via inhibiting the upregulation of HDACs in spinal glial cells. J Neuroinflammation. 2017;14:213. doi:10.1186/ s12974-017-0988-1

17. Chiou CS, Chen CC, Tsai TC, Huang CC, Chou D, Hsu KS. Alleviating bone cancer-induced mechanical hypersensitivity by inhibiting neuronal activity in the anterior cingulate cortex. Anesthesiology. 2016;125:779-792.

doi:10.1097/ ALN.0000000000001237

18. Hua B, Gao Y, Kong X, Yang L, Hou W, Bao Y. New insights of nociceptor sensitization in bone cancer pain. Expert Opin Ther Targets. 2015;19:227-243. doi:10.1517/14728222.2014.980815

19. Zhai M, Yang S, Lin S, et al. Distinct gene expression patterns of ion channels and cytokines in rat primary sensory neurons during development of bone cancer and cancer pain. Front Mol Neurosci. 2021;14:665085. doi:10.3389/fnmol.2021.665085

20. Yuan QL, Guo TM, Liu L, Sun F, Zhang YG. Traditional Chinese medicine for neck pain and low back pain: a systematic review and meta-analysis. PLoS One. 2015;10:e117146. doi:10.1371/journal. pone. 0117146

21. Chou TC, Chang LP, Li CY, Wong CS, Yang SP. The antiinflammatory and analgesic effects of baicalin in carrageenan-evoked thermal hyperalgesia. Anesth Analg. 2003;97:1724-1729. doi:10.1213/01. ANE.0000087066.71572.3F

22. Sun YY, Zhang WJ, Dong CL, et al. Baicalin alleviates nitroglycerin-induced migraine in rats via the trigeminovascular system. Phytother Res. 2017;31:899-905. doi:10.1002/ptr.5811
23. Cherng $\mathrm{CH}$, Lee $\mathrm{KC}$, Chien $\mathrm{CC}$, et al. Baicalin ameliorates neuropathic pain by suppressing HDAC1 expression in the spinal cord of spinal nerve ligation rats. J Formos Med Assoc. 2014;113:513-520. doi:10.1016/j.jfma.2013.04.007

24. Li P, Xiong DL, Sun WP, Xu SY. Effects of baicalin on diabetic neuropathic pain involving transient receptor potential vanilloid 1 in the dorsal root ganglia of rats. Neuroreport. 2018;29:1492-1498. doi:10.1097/WNR.0000000000001138

25. Wang B, Huang T, Fang Q, et al. Bone-protective and anti-tumor effect of baicalin in osteotropic breast cancer via induction of apoptosis. Breast Cancer Res Treat. 2020;184:711-721. doi:10.1007/s10549-020-05904-y

26. Li P, Bi Y, Deng Y, Xiong D, Li A. Baicalin ameliorates bone cancer pain by suppressing TRPV1 in rat dorsal root ganglia. Nat Prod Commun. 2020;15:1934578X19899562. doi:10.1177/ $1934578 X 19899562$

27. Cheng P, Wang T, Li W, et al. Baicalin alleviates lipopolysaccharideinduced liver inflammation in chicken by suppressing TLR4mediated NF-кB pathway. Front Pharmacol. 2017;8:547. doi:10.3389/fphar.2017.00547

28. Min W, Ahmad I, Chang ME, Burns EM, Qian Q, Yusuf N. Baicalin protects keratinocytes from toll-like receptor-4 mediated DNA damage and inflammation following ultraviolet irradiation. Photochem Photobiol. 2015;91(6):1435-1443. doi:10.1111/ php. 12505

29. Zimmermann M. Ethical guidelines for investigations of experimental pain in conscious animals. Pain. 1983;16:109-110. doi:10.1016/ 0304-3959(83)90201-4
Journal of Inflammation Research

\section{Publish your work in this journal}

The Journal of Inflammation Research is an international, peerreviewed open-access journal that welcomes laboratory and clinical findings on the molecular basis, cell biology and pharmacology of inflammation including original research, reviews, symposium reports, hypothesis formation and commentaries on: acute/chronic inflammation; mediators of inflammation; cellular processes; molecular

\section{Dovepress}

mechanisms; pharmacology and novel anti-inflammatory drugs; clinical conditions involving inflammation. The manuscript management system is completely online and includes a very quick and fair peerreview system. Visit http://www.dovepress.com/testimonials.php to read real quotes from published authors. 